\title{
Evaluación de Aprendizajes en Estudiantes con Necesidades Educativas Especiales
}

\section{Evaluation of Learning of Students with Special Educational Needs}

\author{
Karla Muñoz Durán * \\ Maite Otondo Briceño
}

Universidad Católica de la Santísima Concepción, Chile

\begin{abstract}
La evaluación de aprendizajes debe instaurarse como disciplina científica en el ámbito de las Necesidades Educativas Especiales (en adelante NEE). El objetivo de esta investigación fue describir las acciones de evaluación de los aprendizajes para los estudiantes con NEE, realizadas por profesores de aula común y de educación diferencial, evidenciando la coherencia entre estas acciones y la propuesta de la normativa chilena vigente. Se trató de un estudio de integración de paradigmas, secuencial, mixto, por complementación. La primera fase, cuantitativa, implicó un cuestionario aplicado a 107 profesores de la comuna de Concepción, Región del Biobío; para recoger información sobre sus conceptos y acciones. La segunda fase, cualitativa, se desarrolló mediante una entrevista semiestructurada a 12 profesores que participaron de la primera fase, profundizando algunos hallazgos. Como resultado, se encontró diferencias entre los conceptos declarados y las acciones descritas. Los profesores declaran conceptos constructivistas e inclusivos, pero utilizan procedimientos tradicionales, priorizando rendimiento a inclusión; manifiestan realizar acciones según la normativa vigente, pero describen prácticas centradas en un instrumento de evaluación escrito, no incorporando a los padres en la planificación de la evaluación. Algunas conclusiones son: Los profesores priorizan objetivos de aprendizaje, describen trabajo personalizado según características del estudiante; adaptan un instrumento tipo; justifican sus ajustes en base a la calificación y no a características neurológicas o emocionales; el pilar Diseño Universal de Aprendizaje (en adelante DUA) menos instalado tiene relación con el desarrollo de la autonomía, motivación y clima de aceptación en el aula.
\end{abstract}

Palabras clave: Evaluación de los aprendizajes; Necesidades educativas especiales; Programa de integración escolar, Atención a la diversidad.

The evaluation of learning should be established as a scientific discipline within the field of education, including the area of Special Educational Needs (hereafter SEN). The object of this study was to describe the actions of evaluation of learning for students with SEN, carried out by teachers and special educators, demonstrating the coherence between these actions and the current regulation proposed for Chile. This is integration paradigm study, sequential, mixed, complementation. In the first, quantitative phase, a survey was given to 107 teachers in the city of Concepción, Biobío Region, Chile, in order to collect information on the concepts and actions that they carry out. The second, qualitative phase was developed by conducting a semistructured interview with 12 teachers that participated in the first phase, in order to delve into the findings from this stage. The principal findings include the differences between the teachers' declared concepts and described actions. They state constructive and inclusive concepts, but use traditional procedures, prioritizing performance over inclusion; they claim to carry out actions in accordance with the current regulation, but describe practices focused on written tests as the main evaluation instrument, not incorporating parents into the planning of evaluations. The most relevant conclusions show that the surveyed teachers prioritize learning objectives, but in the interview describe personalized work according to the students' characteristics; they adapt one instrument type for all; they justify their adjustments based on the success of the grade and not on neurological or emotional characteristics; the least installed pillar of Universal Design of Learning (hereafter UDL) has to do with the development of autonomy, motivation and an environment of acceptance in the classroom; there is coordination amongst professionals that facilitates decision making, but lack of time for carrying out their ideas.

Keywords: Evaluation of learning; Special educational needs; School integration program; Attention to diversity.

*Contacto: maite@ucsc.cl

issn: 1989-0397

www.rinace.net/riee/

https://revistas.uam.es/riee
Recibido:

19 de junio de 2018

$1^{\text {a }}$ Evaluación: 10 de julio de 2018

Aceptado: $\quad 20$ de agosto de 2018 


\section{Introducción y presentación del problema}

El sistema educativo chileno se ha reformado profundamente, garantizando educación a todos los estudiantes, para su acceso, participación y progreso; intentando asegurar calidad en la educación pública. Se incorporan apoyos para estudiantes con NEE, preocupación explícita en la Ley General de Educación (en adelante LGE), señalando que la educación debe propender a garantizar que todos los estudiantes, independiente de sus condiciones y circunstancias, alcancen objetivos generales y estándares de aprendizaje (Ley 20.370, 2009), además el sistema propenderá a cautelar que todos tengan las mismas oportunidades de recibir una educación de calidad, sobre todo aquellas personas o grupos que requieran apoyo especial (Ley nº 20.370, 2009). También, se regula la subvención de estudiantes pertenecientes a Programas de Integración Escolar (en adelante PIE) (Ley 20.201, 2007); las Orientaciones Técnicas para PIE (MINEDUC, 2013); y los criterios y adecuaciones curriculares para los estudiantes con NEE (Decreto exento $\mathrm{n}^{\circ}$ 83, 2015). Estos documentos legales han sido difundidos por el Ministerio de Educación (en adelante MINEDUC) asegurando que profesores de aula común y diferencial trabajen juntos, garantizando el desarrollo de aprendizajes y la evaluación, de alumnos con NEE. La concreción de este proceso no puede asegurarse sin dar cuenta en la evaluación el grado de aprendizaje alcanzado por los estudiantes.

Se observa ambigüedad en la definición de papeles y responsabilidades en la evaluación de los avances y resultados de aprendizaje (López, Julio, Pérez, Morales y Rojas, 2014), además de una distancia entre el discurso inclusivo MINEDUC y las prácticas evaluativas efectivamente implementadas (Marfán, Castillo, González y Ferreira, 2013), que podría estar relacionado con que la política educativa chilena propone la inclusión desde los derechos humanos, pero prescribe un modelo de integración de énfasis psicomédico (López et al., 2014); donde la ley determina los requisitos, instrumentos, pruebas diagnósticas para elegir a los beneficiarios de una subvención (Peña, 2013). El diagnóstico tendrá un rol fundamental en la escuela para el funcionamiento correcto y fluido del aprendizaje (Реп̃a, 2013).

Dados estos antecedentes, se observa un vacío de conocimiento y necesidad de investigación sobre las acciones realizadas por profesores para la evaluación de los aprendizajes en estudiantes con NEE transitorias y permanentes, investigando las prácticas de adecuación evaluativa implementadas por profesores de aula común y diferencial, en coherencia con las premisas de MINEDUC, en función de la calidad, progreso y promoción de todos los estudiantes dentro del sistema educativo chileno.

\section{Marco conceptual o teórico de la investigación}

\subsection{Evaluación de los aprendizajes}

La evaluación de los aprendizajes es entendida como "proceso de delinear, obtener, procesar y proveer información válida, confiable y oportuna que permita juzgar el mérito o valía de programas, procedimientos y productos con el fin de tomar decisiones de mejora” (Ahumada, 2001, p. 22). 
Se observan diversos enfoques evaluativos, encontrándose (a) normas, (b) criterios, (c) construcción de conocimientos, en función de un aprendizaje significativo y (d) enfoque auténtico, de enseñanza personalizada y evaluación diferenciada (Ahumada, 2005), incorporando una mirada inclusiva, individualizada y multidimensional.

Con respecto a los momentos e intencionalidad de la evaluación de los aprendizajes, se puede señalar tres grandes instancias: (a) evaluación inicial se relaciona con la evaluación diagnóstica, que busca conocer el aspecto personal y académico; se realiza al inicio de una unidad de aprendizaje (Ahumada, 2005). La (b) evaluación procesual, identifica fortalezas de los alumnos y muestra errores o falencias del quehacer pedagógico; implica reflexión docente, para realizar modificaciones didácticas (Muñoz, 2006). Por último, la (c) evaluación final se realiza en función de propósitos establecidos, para acreditar aprendizajes alcanzados (Muñoz, 2006). Busca valorar el logro de los objetivos, comprobar el desarrollo de las capacidades que los alumnos trabajaron, posibilita la reflexión. Al igual que la Evaluación Sumativa, es un proceso selectivo, certifica el nivel de logro de un aprendizaje asignándole una calificación, mediante la demostración de competencias en una situación real o simulada. Se realizará al finalizar una unidad de aprendizaje (Ahumada, 2005).

Antes de desarrollar un procedimiento o instrumento de evaluación, deben considerarse el proceso en el que se inscriben todas las acciones que conlleva. Esta estrategia de evaluación se refiere a un plan que incluye el momento y los instrumentos necesarios para facilitar la evaluación (Llanos y Escamilla, 1995). Dentro de este plan, también debe considerarse el proceso de construcción del aprendizaje y el tipo de contenido que se quiere evaluar (Ahumada, 2001), en función de los procedimientos e instrumentos más adecuados.

Para planificar y diseñar procedimientos de evaluación adecuados, Ahumada (2001) diferencia entre la evaluación de contenidos (a) declarativos, terminologías, hechos, enunciación de teorías, leyes o principios que serán recordados; (b) procedimentales relacionados con actividades de ejecución manual (diseñar un plano, mapa o confección de una maqueta) y con aquellas que implican acciones y decisiones cognitivas (recopilación y organización de información, resolución de problemas); finalmente, para evaluar contenidos (c) actitudinales, considera lo que el estudiante conoce, lo que siente o prefiere, y las acciones que manifiesta en torno al tema.

En relación con los instrumentos de evaluación, tradicionalmente se determinan conocimientos de contenidos declarativos con pruebas escritas y orales, tipo cuestionario. Los aprendizajes procedimentales y actitudinales se registran en (a) lista de cotejo, fácil de diseñar y aplicar, o (b) escala de apreciación, más objetivas, pero más compleja. De acuerdo con Ahumada (2001), estos instrumentos tradicionales y uniformes, impiden la utilización de diversas situaciones educativas para conocer los niveles de logro; por esto propone un sistema de evaluación alternativa, con instrumentos no tradicionales divergentes, centrados en qué y cómo aprende el estudiante, obligándole a responsabilizarse del proceso. Para los contenidos conceptuales, propone mapa conceptual, mapa semántico y diagrama de síntesis. Para los contenidos procedimentales, puede utilizarse pauta de observación, autoinforme y co-evaluación.

Para evaluar contenidos actitudinales, Yus (1996, citado en Ahumada, 2001) diferencia entre métodos no observacionales (cuestionarios, encuestas y pautas de cotejo) y métodos 
observacionales narrativos (registro anecdótico, diarios de clase, registros de audio y video, cuestionarios de co-evaluación y auto-observación).

\title{
2.2. Educación especial
}

Al finalizar los años setenta, el Informe Warnock, asocia la educación a un derecho para todos, introduciendo el concepto de NEE. La Unesco define educación especial como "una forma enriquecida de educación general tendiente a mejorar la vida de aquellos que sufren diversas minusvalías, enriquecida en el sentido de recurrir a métodos pedagógicos modernos y al material técnico para remediar ciertos tipos de deficiencias" (Unesco, 1977, p. 25 en Castejón y Navas, 2009).

En la década de los ochentas, la Organización Mundial de la Salud (OMS) publica la Clasificación Internacional del Funcionamiento, la Discapacidad y la Salud (CIF), actualizando el concepto de discapacidad, como "limitaciones en la actividad y restricciones en la participación. Indica los aspectos negativos de la interacción entre un individuo (con una condición de salud) y sus factores contextuales (factores ambientales y personales)" (Organización Mundial de la Salud, 2001, p. 206). En Chile, en 1989 se decretan planes y programas de estudios específicos para cada NEE, de acuerdo a sus características, descontextualizándolos del plan común (Echeita, 2006).

En 1994, la Unesco redefine educación especial formulando las condiciones de una escuela para todos, donde el sistema escolar de un país debe ajustarse para satisfacer las necesidades de todos (Unesco, 1994, citado en Echeita, 2006). Actualmente, en Chile la educación especial es entendida como una:

\begin{abstract}
Modalidad del sistema escolar que provee servicios y recursos especializados, tanto a los establecimientos de enseñanza regular como a las escuelas especiales, con el propósito de asegurar, de acuerdo a la normativa vigente, aprendizajes de calidad a niños, niñas y jóvenes con necesidades educativas especiales asociadas o no a una discapacidad, asegurando el cumplimiento del principio de igualdad de oportunidades para todos los educandos. (Ley 20.422, 2010, p.19).
\end{abstract}

\subsection{Marco Normativo}

Durante la última década Chile ha realizado modificaciones legales como respuesta educativa a la diversidad. En 1998 el Decreto de ley $\mathrm{N}^{\circ} 1$ entrega disposiciones mínimas para la atención de estudiantes con NEE, originando la modalidad de integración (Decreto 1, 1998) creó conciencia en la comunidad educativa de la existencia de las NEE y su derecho de acceder a una educación igualitaria.

Promulgada el año 2007, la Ley 20.201 que modifica subvenciones a establecimientos educacionales, define el concepto de NEE Transitorias y NEE Permanente, anticipando un reglamento, Decreto con toma de razón $\mathrm{N}^{\circ} 170$, que fija las normas para determinar los alumnos que serán beneficiarios de las subvenciones para educación especial (Decreto 170, 2009), y establece criterios diagnósticos de los estudiantes que pueden ser beneficiarios.

Meses más tarde, en 2009 se publica la Ley 20.370 General de Educación, que regula los derechos y deberes de los integrantes de la comunidad educativa, con el objetivo de alcanzar "un sistema educativo alcanzado por la equidad y calidad de su servicio" (Ley 20.370, 2009, p. 1). Define educación como un proceso de aprendizaje permanente que abarca las distintas etapas de la vida de las personas, para alcanzar su desarrollo espiritual, 
ético, moral, afectivo, intelectual, artístico y físico, mediante la transmisión y el cultivo de valores, conocimientos y destrezas; enmarcado en la valoración y el respeto de los derechos humanos y la diversidad cultural, para que las personas logren conducir su vida en forma plena, convivir y participar (Ley 20.370, 2009). En su artículo tercero, declara sus intenciones de equidad mediante principios; entre los cuales se encuentran (a) Universalidad y educación permanente para que la educación esté al alcance de todas las personas, (b) calidad de la educación independiente de las condiciones y circunstancias, (c) equidad para asegurar que todos tengan las mismas oportunidades, (d) respeto de la diversidad cultural, religiosa y social, (e) participación a todos los miembros de la comunidad, e (f) integración para la incorporación de alumnos de diversas condiciones sociales, étnicas, religiosas, económicas y culturales (Ley 20.370, 2009; p. 2). Se incorpora la Agencia de Calidad de la Educación que "evaluará los logros de aprendizajes de los alumnos y el desempeño de los establecimientos, de acuerdo a estándares indicativos” (Ley 20.370, 2009, p.3).

La LGE incorpora criterios inclusivos, como el derecho a recibir una educación que "ofrezca oportunidades de formación y desarrollo integral", recibir una "atención adecuada y oportuna” en el caso de las NEE y a no ser discriminados. (Ley 20.370, 2009, p.4); pero no cambia el sistema tripartito, concentrando estos estudiantes en establecimientos particulares subvencionados y, principalmente aquellos con NEE de tipo permanente, en establecimientos públicos.

El año 2010, se cambia la legislación sobre integración social de personas con discapacidad por otra que se "adecue a los nuevos paradigmas, a los instrumentos jurídicos internacionales y a los avances del derecho comparado para hacer efectivo el derecho a la igualdad de oportunidades de las personas con discapacidad" (Biblioteca del Congreso Nacional de Chile, 2010, p. 17). Para ello se promulga la Ley 20.422 que Establece normas sobre igualdad de oportunidades e inclusión social de personas con discapacidad, redefiniendo conceptos como igualdad de oportunidades, que es abordada como "ausencia de discriminación por razón de discapacidad, así como la adopción de medidas de acción positiva orientadas a evitar o compensar las desventajas de una persona con discapacidad para participar plenamente en la vida política, educacional, laboral, económica, cultural y social” (Ley 20.422, 2010, p. 5). En cuanto a la educación, indica que "el Estado garantizará a las personas con discapacidad el acceso a los establecimientos públicos y privados del sistema de educación regular o a los establecimientos de educación especial, según corresponda, que reciban subvenciones o aportes del Estado" (Ley 20.422, 2010, p. 19); asegurando que las escuelas comunes incorporarán innovaciones y adecuaciones curriculares, de infraestructura y los materiales de apoyo necesarios para permitir y facilitar a las personas con discapacidad el acceso, brindándoles recursos adicionales para asegurar su permanencia y progresso (Ley 20.422, 2010).

En relación a la evaluación, la Ley 20.422 señala que "Los instrumentos que se utilicen para la aplicación de mediciones de la calidad de la educación deberán contemplar las adecuaciones necesarias para que los alumnos y alumnas con necesidades educativas especiales puedan participar" (Ley 20.422, 2010, p. 36).

El MINEDUC emite Orientaciones Técnicas para PIE, que "regula los requisitos, procedimientos y pruebas diagnósticas, así como los profesionales competentes, para evaluar a estudiantes que presentan NEE de carácter permanente (asociados a una 
discapacidad), o NEE de carácter transitorio" (MINEDUC, 2013a, p. 22). Define evaluación interdisciplinaria como una indagación sobre NEE en un estudiante, realizada por profesionales de la salud y del área psicoeducativa, "a fin de determinar los apoyos especializados que el estudiante requiera para participar y progresar en su desarrollo y aprendizaje" (MINEDUC, 2013a, p. 23).

Este mismo documento especifica para la evaluación de aprendizajes, cuatro puntos para obtener información clave, decidiendo las metas educativas, determinar y modificar estrategias a utilizar en el contexto escolar y familiar para lograr los aprendizajes esperados y conseguir una participación efectiva en la comunidad educativa: (a) aprendizajes logrados, (b) estilo de aprendizaje y motivación, (c) aspectos del desarrollo personal y social, (d) capacidades, fortalezas y dificultades (MINEDUC, 2013).

El equipo multidisciplinario, debe tomar decisiones referidas a la evaluación de aprendizajes de los estudiantes que presentan NEE transitorias y permanentes, tales como (a) Decidir modalidades, procedimientos e instrumentos de evaluación, que se ajusten a la comprensión, expresión y ejecución de los estudiantes; (b) Uso flexible de los tiempos de evaluación, respetando ritmos de aprendizaje y de ejecución; (c) Utilizar la información de la evaluación inicial para determinar quiénes requieren una evaluación interdisciplinaria y quiénes necesitan adecuaciones curriculares, favoreciendo su progreso en el currículum escolar y la participación en el nivel; (d) Convenir diversos procedimientos de evaluación de los aprendizajes, como: mayor tiempo, diversos momentos de la evaluación, adecuaciones para acceder a los instrumentos de evaluación, entre otros; y (e) Concordar la conveniencia de registrar los resultados de evaluación de cada estudiante en la plataforma informática. (MINEDUC, 2013).

Recientemente entra en vigencia el Decreto exento 83 que aprueba criterios y orientaciones de adecuación curricular para estudiantes con NEE de educación parvularia y educación básica; entendiendo una transición en la forma de comprender las dificultades del aprendizaje, desde un modelo centrado en el déficit hacia un enfoque educativo que implica el desarrollo integral de las características individuales de los estudiantes. "Establece criterios y orientaciones para realizar adecuaciones curriculares que permitan planificar propuestas educativas de calidad a los estudiantes que lo requieran" (Decreto 83 exento, 2015, p. 3).

\section{Marco Referencial}

En el contexto de fuertes cambios normativos, se han realizado diversas investigaciones tendientes a observar el grado de implementación de la política relativa a PIE y las condiciones de la escuela para atender a la diversidad.

El estudio Barreras Culturales para la Inclusión (López et al., 2014), analiza las concepciones y prácticas sobre integración, a fin de observar la relación entre la política educativa y las prácticas locales. Al momento estudiado, la política sobre integración era optativa y complementaria; no declarándose una relación entre el aprendizaje de los estudiantes que participan en PIE y otros alunos (López et al., 2014). La atención y aprendizaje de estudiantes con NEE era responsabilidad de docentes especialistas, externalizando las obligaciones educativas, desde el profesor de aula regular hacia el 
docente de educación diferencial (López et al., 2014). También se atribuye la responsabilidad del aprendizaje al mismo estudiante NEE y su familia, haciendo que docentes de aula común y diferencial pierdan su compromiso con sus responsabilidades en el aprendizaje. Esta investigación detecta que los tiempos de planificación de la codocencia se destinaban a mejorar el rendimiento académico y no a analizar temáticas sobre la integración.

Entre las conclusiones del estudio se observa que (a) aunque la política se refiere a la diversidad desde la inclusión, se centra en un "modelo médico-clínico, que descansa sobre la visión patológica de la diferencia” (López et al., 2014, p. 276) por lo que el aprendizaje de estudiantes NEE dependen de su discapacidad, del apoyo familiar y su cultura de origen. (b) El financiamiento se realiza a través de la categorización diagnóstica para recibir subvención especial; reforzando la segregación y discriminación en la escuela (Echeita, 2007 em (López et al., 2014), aumentando el diagnóstico de NEE (Graham y Jahnukainen, 2011 en López et al., 2014). (c) El decreto 170 no tiende a la inclusión, sino que pone en riesgo la equidad educativa al aumentar la segregación (Ferguson, 2008 en López et al., 2014). Finalmente se observa que la política educativa, condiciona la entrega de recursos adicionales, creando estándares nacionales que conforman barreras para la inclusión, dado que conllevará a la formación de jerarquías de valoraciones de estudiantes (Graham y Jahnukainen, 2011 en López et al., 2014).

En 2012, el Centro de Innovación en Educación de Fundación Chile, por solicitud del MINEDUC, realiza un Análisis de la Implementación de los PIE; para describir la instalación de la política PIE y la situación actual de las escuelas, en sus procesos escolares y experiencias de aprendizaje. Este estudio señala la región del Biobío como la de mayor concentración de escuelas PIE, mayor a la región metropolitana a pesar de su demografía; esto se relaciona con que escuelas municipales como particular subvencionadas han incorporado la política PIE (Marfán et al., 2013), llegando a tener un promedio de cuarenta alumnos en integración por cada escuela (Marfán et al., 2013). En las regiones del Maule, Biobío y Araucanía, la mayoría de escuelas con PIE pertenecen al segmento socioeconómico bajo, "lo que determina una realidad mucho más compleja que en otras regiones del país, la que sumada a problemas de segregación, ruralidad y vulnerabilidad demanda más y mejores herramientas para trabajar temas de integración y diversidad" (Marfán et al., 2013, p.95). El Índice de Vulnerabilidad Escolar (IVE) más bajo se concentra en las escuelas de dependencia municipal, destacándose estadísticamente la región del Biobío al observar 16,3 puntos porcentuales de IVE adicionales en las escuelas rurales que en las urbanas (Marfán et al., 2013). Además, el grupo socioeconómico bajo posee mayor cantidad de NEE permanentes (NEEP) y la región del Biobío presenta la mayor cantidad de estudiantes con NEEP en el país (Marfán et al., 2013).

La investigación observó que las escuelas cuentan con valores de integración e inclusión "sin embargo en la práctica lo más integrativo es la existencia de PIE y en la mayoría de los casos, la no existencia de criterios de selección en los estudiantes" (Marfán et al., 2013, p. 159). La Evaluación Diferenciada es un tema complejo de realizar, pues "existe declaración de voluntad, pero en la práctica es una acción llevada a cabo con muchas dificultades o resistencias, en todos los establecimientos estudiados" (Marfán et al., 2013, p. 160). El trabajo colaborativo, está poco formalizado, recae en los profesionales PIE generar instancias de trabajo y en los profesores de aula regular el tener disposición (Marfán et al., 2013). Las actitudes y prácticas de las escuelas tienden más hacia la 
integración que a la inclusión. Se trata a los niños como si fueran iguales, pero se les integra desde un diagnóstico, no se trabaja para superar barreras.

Entre sus conclusiones, se observa que (a) existe dualidad en la instalación de procesos asociados a PIE, no existe el mismo nivel de institucionalización con respecto a una lógica de trabajo que asegure aprendizaje de calidad para todos los estudiantes; (b) las escuelas cumplen con requisitos mínimos de diagnósticos y profesionales involucrados en este proceso; (c) se observan desafíos por desarrollar prácticas de institucionalización de la política PIE, pues existe debilidad en la organización y planificación de acciones para el trabajo con la diversidad al interior del aula; (d) las escuelas señalan trabajar con flexibilización curricular y evaluación diferenciada, pero no se observó existencia de un trabajo profundo al respecto, declarando voluntad de realizar evaluación diferenciada pero observándose dificultades como falta de tiempo y conocimiento para realizarlas, además de contraposición con rendimiento exitoso en pruebas de medición; (e) al no discutir en relación a los contenidos, metodologías y modos de evaluación, se debilitan las acciones de trabajo colaborativo en aula y el involucramiento mutuo de los docentes.

La investigación Atención a la Diversidad en el contexto chileno (San Martín, 2012) describe concepciones docentes respecto a dilemas de diseño de la propuesta curricular y evaluativa para atender a la diversidad; principalmente NEEP. Contiene un apartado específico sobre evaluación, considerándola como "la forma en que los profesores y otras personas implicadas en la educación sistemática de un alumno recogen y emplean la información sobre los logros alcanzados y/o desarrollados en las distintas áreas de su experiencia educativa" (European Agency for Developent in Specials Needs Education, 2007, citado en San Martín, 2012, pp. 168-169); entendiendo por estas áreas la académica, conductual y social. El estudio observa una tendencia a la evaluación según modelo clásico y sumativo, que asegura el acceso a la información objetiva, pero tiende a homogeneizar los niveles de aprendizaje para el alumnado, concentrándose en exámenes idénticos para todos.

Se encontraron dificultades para la evaluación de los aprendizajes en alumnos con NEE permanentes y su promoción. Los profesores de educación general básica (en adelante EGB) señalan la necesidad de realizar una evaluación unificada y homogénea para todos; mientras que los profesores de educación diferencial indican errores conceptuales en torno a la evaluación diferenciada, que es considerada por los profesores EGB como disminución de la complejidad y del número de ítems de un instrumento. También indicaron que los procesos evaluativos deben considerar las características, habilidades y necesidades de todo el alumnado, adecuando los niveles de logro a cada niño. En cuanto a la promoción y evaluación, se señala una falta de formación profesional y desconocimiento de estrategias para la evaluación de alumnos con NEE permanentes, además de requerir tiempo y trabajo adicional para el docente. Finalmente, los profesores EGB indican que los profesores diferenciales tienen tiempo y herramientas para llevar a cabo los procesos evaluativos, asignándoles la responsabilidad de determinar las calificaciones del alumnado con NEE permanentes.

Realizada en 2010, por la Universidad Central en colaboración con MINEDUC, la investigación Flexibilización del Currículum para Atender a la Diversidad, propone criterios y orientaciones de flexibilización del currículo para atender a la diversidad. 
Constó tres fases: (a) análisis documental de la normativa curricular chilena, (b) consulta a expertos internacionales para recoger visiones, enfoques y modelos que aseguren el acceso y la participación de estudiantes con NEE y (c) elaboración de una propuesta revisada por la Unidad de Educación Especial y Unidad de Currículum del MINEDUC. Aborda la evaluación como un proceso articulado con la acción educativa, entregando una retroalimentación para la toma de decisiones futuras y, si es auténticamente continua, facilitará una mirada longitudinal del proceso de aprendizaje y la práctica docente, pudiéndose verificar los progresos y los factores que lo afecten (Duk y Loren, 2010). Se indica la importancia de las decisiones relativas a la evaluación, para dar respuesta a la diversidad; pues afectarán el tipo de información recogida, el modo y el momento en que esto se realiza. El objetivo de planificar la evaluación aseguraría que esta entregue claridad de la situación inicial de cada alumno en particular, su progreso en función a las metas establecidas, a sus conocimientos y habilidades previas y a los objetivos que se planificaron para ese estudiante en particular (Duk y Loren, 2010). También señala que la adaptación curricular facilitará la participación y logros de aprendizajes de todos los estudiantes; no obstante, para aquellos estudiantes que al no ofrecerles una respuesta ajustada podrían fracasar o desertar del sistema escolar, se debe realizar una diversificación curricular, que implicará realizar cambios en la organización de la evaluación. Dentro de la evaluación inicial del alumnado, para determinar sus NEE, habilidades y potencialidades, se mencionan como indicadores de alerta factores del contexto escolar, como los programas de estudio y sistemas de evaluación rígidos (Duk y Loren, 2010).

\section{Metodología}

La investigación se enmarca en la modalidad de integración de paradigmas por complementación, secuencial, mixto. En una primera fase, se especifican objetivos y preguntas de investigación cuantitativos, se realiza una revisión de la literatura para la construcción de un marco teórico base, para establecer hipótesis y variables, además de un plan para probarlas (Hernández, Fernández y Baptista, 2014), con el fin de describir datos reales, posibles de registrar y cuantificar para su análisis, en un contexto real que corresponde a la comuna de Concepción, Región del Biobío, Chile. Este estudio descriptivo, no experimental, transeccional, se realizó a través de un cuestionario de 21 preguntas cerradas, validado por juicio de expertos, autoadministrado a una muestra intencionada de 107 participantes, correspondiente a profesores de educación básica, enseñanza media, educación diferencial y educadoras de párvulos, que se desempeñaban en establecimientos con PIE de la comuna de Concepción, que trabajaban directamente con estudiantes con NEE y que además, realizaban un trabajo de coordinación para la atención de estos alumnos; con el fin de recoger información sobre los conceptos y las acciones que realizan. Las variables que se desprenden de los objetivos son las Acciones de Evaluación de los Aprendizajes y Acciones de Atención a la Diversidad.

En la fase cuantitativa, la muestra fue intencionada y se delimitó a profesores, de educación básica y enseñanza media, profesores de educación diferencial y educadoras de párvulos, que se desempeñaban en establecimientos con PIE de la comuna de Concepción, que trabajaban directamente con estudiantes con NEE y que, además, realizaban un trabajo de coordinación para la atención de estos alumnos. Fue no probabilística, pues "los datos no podrán extrapolarse a toda la población” (Buendía, Colás y Hernández 1998, p. 143). 
Corresponden a profesores de educación diferencial (48\%) y educación general básica $(38 \%)$. De un total de 107 participantes, 32 trabajan en el sistema particular subvencionado y 75 en el sistema municipal.

Cuadro 1. Ejemplo de la definición conceptual de las variables de cuestionario

\begin{tabular}{ll}
\hline \multicolumn{1}{c}{ VARIABLE } & \multicolumn{1}{c}{ DEFINICIÓN CONCEPTUAL } \\
\hline & $\begin{array}{l}\text { Evaluación de los aprendizajes: Proceso para delinear, obtener, } \\
\text { procesar y proveer información válida, confiable y oportuna que } \\
\text { permita juzgar el mérito o valía de programas, procedimientos y }\end{array}$ \\
$\begin{array}{l}\text { Acciones de evaluación } \\
\text { de los aprendizajes }\end{array}$ & $\begin{array}{l}\text { productos con el fin de tomar decisiones de mejora (Ahumada, 2001, } \\
\text { El Enfoque Evaluativo: Modelo educativo que puede estar centrado } \\
\text { en: Normas, criterios, construcción de conocimientos, enfoque }\end{array}$ \\
& $\begin{array}{l}\text { Auténtico, (Ahumada, 2005, p.31). } \\
\text { Acciones de evaluación }\end{array}$ \\
de los aprendizajes & $\begin{array}{l}\text { obtentos de la Evaluación: Partes del proceso educativo que permiten } \\
\text { Antencionalidad de la Evaluación: Objetivo del proceso educativo en } \\
\text { Acciones de evaluación } \\
\text { de los aprendizajes }\end{array}$ \\
& $\begin{array}{l}\text { relación a conocer al estudiante, descubrir las causas de sus errores y } \\
\text { juicio valorativo (Barberá, 1999; Castillo y Cabrerizo, 2003). }\end{array}$ \\
\hline
\end{tabular}

Fuente: Elaboración propia.

Participaron profesores de 18 escuelas $(81,81 \%)$ que imparten enseñanza básica de administración municipal de la comuna de Concepción. Los profesores del sistema municipal corresponden al 22,05\% de una población del profesorado que realiza trabajo coordinado para la atención de alumnos con NEE, incorporados a PIE.

La segunda fase, cualitativa, complementó la información y profundizó algunos de los hallazgos, con el fin de entender mejor el fenómeno estudiado (Bericat, 1998). Se aplicó una entrevista semiestructurada, a 12 profesores que participaron de la primera fase y se les invitó a participar de una entrevista en forma voluntaria (Hernández et al., 2014). Las profesionales corresponden a dos profesoras diferenciales, dos educadoras de párvulos y ocho profesoras de educación general básica (en adelante EGB), dos con perfeccionamiento en educación diferencial, dos con especialidad en lenguaje de segundo ciclo básico, tres con especialidad matemática de segundo ciclo básico, y una con especialidad en lenguaje e historia. En esta entrevista se consultó a los docentes por las acciones que realizan para la adecuación de la evaluación de los aprendizajes, los pasos que requieren esas acciones, las modificaciones que requieren los instrumentos de estudiantes NEE y quiénes participan de todo ese proceso; enfatizando en que el relato fuera direccionado desde su experiencia docente y práctica profesional; con el fin de complementar los hallazgos de la primera fase de investigación.

\section{Resultados}

\subsection{Fase cuantitativa}

En la fase cuantitativa se realizó un análisis de homogeneidad a través del paquete Homals de $\mathrm{R}$, que mezcla dos tipos de análisis: el análisis de múltiples correspondencias, que representa un conjunto de respuestas categóricas como respuestas numéricas, asignando valores a los ítems y a las personas, de manera que las agrupa de acuerdo al parecido de 
sus respuestas; por otra parte, el escalamiento óptimo, que permite transformar los puntos asignados a las personas y sus respuestas de acuerdo al nivel de medición de la variable.

Se consideró cada respuesta como una variable nominal. Al realizar el análisis Homals, se asignó a cada docente un puntaje dentro de una sola variable que permitió entender su forma de responder todas las preguntas, correspondiente a un determinado enfoque de evaluación de los aprendizajes. En los puntos extremos de este puntaje, se encontraron dos grupos definidos: por una parte un grupo parece acercarse a un enfoque tradicional de la evaluación como una valoración del conocimiento, con una mirada centrada en analizar el logro de objetivos en un estudiante; por otro lado, se observan docentes cercanos a un enfoque constructivista de la evaluación, como proceso que permite tomar decisiones y realizar cambios didácticos, con una mirada centrada en construir aprendizajes nuevos en torno a aprendizajes significativos de los estudiantes.

En las preguntas sobre evaluación de los aprendizajes, la mayoría de los docentes consultados coincide que se trata de un proceso que permite tomar decisiones y realizar cambios didácticos; se centran en construir aprendizajes nuevos en torno a los aprendizajes significativos de los estudiantes; no priorizan ningún momento de la evaluación; coinciden en que los tipos de evaluación según intencionalidad tienen igual importancia con respecto al trabajo con NEE; entiende por estrategia evaluativa el momento y los instrumentos necesarios para planificarla; los procedimientos de evaluación más utilizados son (a) recopilación y organización de la información, (b) preguntas previas, (c) lluvia de ideas y (d) organizadores gráficos; los instrumentos de evaluación que más se repiten son (a) prueba escrita, (b) pauta de observación e (c) interrogación oral.

En las preguntas sobre educación especial, los profesionales señalan que el PIE es un sistema que genera las condiciones para satisfacer las necesidades de todo el grupo de estudiantes; más de la mitad de los profesionales participantes señalan que la inclusión es manifestada dentro del establecimiento educacional como presencia, participación y rendimiento de todos los alumnos sin distinción; más de la mitad de los participantes relacionan con las NEE con necesidades individuales que requieren ser atendidas mediante una serie de apoyos especiales.

Para la atención de estudiantes con NEE al interior de los establecimientos, los ajustes más realizados corresponden a (a) priorizar objetivos de aprendizaje, (b) modificar tipo y grado de aprendizaje a conseguir, (c) temporalizar objetivos o criterios de evaluación; dan cuenta de que el modelo más utilizado para la planificación de actividades para sus alumnos con NEE, es el plan de adecuación curricular individual (en adelante PACI). A lo menos participan tres integrantes en la toma de decisiones; los dos primeros están bien definidos y corresponden a (a) profesor de aula común y (b) profesor diferencial.

Como comunidad educativa se han organizado para alcanzar de forma real a lo menos dos de los principios presentados; observándose menor cantidad de respuestas para el principio de universalidad y educación permanente para que la educación esté al alcance de todas las personas. Obtienen información desde los aprendizajes logrados, estilos de aprendizaje y motivación, aspectos del desarrollo personal y social, y las capacidades individuales. La función más realizada por los equipos de aula es identificar fortalezas y debilidades del grupo curso. Las acciones más realizadas son tomar decisiones respecto de las modalidades, procedimientos e instrumentos de evaluación ajustados a las 
posibilidades de comprensión, expresión y ejecución de los alumnos. La acción menos reiterada corresponde a definir la conveniencia de diversos procedimientos de evaluación. El principio DUA menos instalado es la tercera acción: Proporcionar múltiples medios de compromiso ofreciendo opciones que reflejen los intereses de los alumnos. En relación con la instalación de la inclusión, coinciden en encontrarse en la fase 1: Equipo responsable del plan de mejora educativa organiza un grupo de coordinación.

\subsection{Fase cualitativa}

En la fase cualitativa, se presentan los resultados obtenidos mediante un análisis del contenido (Pérez, 2011), técnica utilizada para el análisis sistemático de, en este caso, entrevistas semiestructuradas registradas; para ello se realiza, en primera instancia, un análisis del contenido del discurso, que permitió realizar una codificación de la información entregada por las profesoras participantes, para luego describir cada una de estas. Se establecieron códigos descriptivos (Pérez, 2011) para determinar la frecuencia de un contenido a un segmento de las respuestas entre las docentes. Posteriormente se presenta la descripción de cada una de las categorías que se levantaron a partir de los códigos identificados en la etapa anterior, los que corresponden a las cuatro categorías contempladas en el guión temático; no obstante esta descripción de categorías, se utiliza la técnica de las comparaciones constantes, consistente en codificar las categorías propuestas, comparándolas con los incidentes específicos observados, lo que permite una reelaboración de categorias (Pérez, 2011).

En este caso, tras realizar el análisis del discurso, se encuentran nuevas subcategorías, que permiten explicar mejor cada una de las categorías. Finalmente se desarrolló una matriz de triangulación de acuerdo con el área de especialización de las docentes.

Cuadro 2. Matriz de objetivos, categorías, subcategoría y pregunta de guión entrevista semi estructurada

\begin{tabular}{|c|c|c|c|}
\hline $\begin{array}{c}\text { OBJETIVO } \\
\text { ESPECÍFICO } \\
\end{array}$ & CATEGORÍA & SUBCATEGoRÍA & Pregunta \\
\hline \multirow{5}{*}{$\begin{array}{l}\text { Indagar la } \\
\text { experiencia de } \\
\text { los profesores } \\
\text { respecto a la } \\
\text { adaptación } \\
\text { evaluativa que } \\
\text { requieren sus } \\
\text { estudiantes con } \\
\text { NEE }\end{array}$} & \multirow{5}{*}{$\begin{array}{c}\text { Experiencias } \\
\text { de } \\
\text { adaptación } \\
\text { en la } \\
\text { evaluación }\end{array}$} & Acciones & $\begin{array}{l}\text { Según su experiencia ¿Cuáles son las } \\
\text { acciones evaluativas involucradas en la } \\
\text { evaluación de alumnos con NEE? }\end{array}$ \\
\hline & & $\begin{array}{l}\text { Acciones de } \\
\text { proceso }\end{array}$ & $\begin{array}{l}\text { Actualmente ¿Cómo realiza el proceso } \\
\text { para evaluar los aprendizajes de sus } \\
\text { estudiantes con NEE? ¿Qué significa esto? }\end{array}$ \\
\hline & & $\begin{array}{l}\text { Adaptaciones de } \\
\text { evaluación } \\
\text { declaradas }\end{array}$ & $\begin{array}{l}\text { (Continuación) ¿Qué debe considerar a la } \\
\text { hora de evaluar un alumno TEA? ¿y uno } \\
\text { FIL? }\end{array}$ \\
\hline & & $\begin{array}{l}\text { Instrumentos } \\
\text { utilizados }\end{array}$ & $\begin{array}{l}\text { Le voy a contar un caso ¿Usted tiene } \\
\text { algún caso como Pablo? ¿Qué } \\
\text { instrumentos utiliza para evaluar sus } \\
\text { aprendizajes? } \\
\text { ¿A qué atribuye que muy pocos profesores } \\
\text { utilicen instrumentos como portafolios, } \\
\text { entrevistas, dramatizaciones, proyectos de } \\
\text { aula, para evaluar a sus estudiantes NEE? }\end{array}$ \\
\hline & & $\begin{array}{c}\text { Adecuaciones al } \\
\text { instrumento de } \\
\text { evaluación }\end{array}$ & $\begin{array}{l}\text { ¿Qué ajustes usted hace a sus } \\
\text { instrumentos de evaluación? }\end{array}$ \\
\hline
\end{tabular}

Fuente: Elaboración propia en base a 12 entrevistas. 
Entre los principales resultados se observa que la mayoría de las docentes hace referencia a la adecuación de la evaluación como la principal acción evaluativa que se debe realizar para estudiantes con NEE; las educadoras de párvulos verbalizan realizar ajustes como trabajo personalizado y apoyo de material concreto, acciones que sí corresponden a ajustes de acceso a la evaluación (Decreto exento $\mathrm{n}^{\circ}$ 83, 2015); por lo tanto es posible decir que realizan evaluación diferenciada, pero no han internalizado la dimensión de este concepto.

Cuadro 3. Ejemplo del Listado de códigos de entrevista semi estructurada

\begin{tabular}{|c|c|c|c|}
\hline CódIGO & DESCRIPCIÓN & SIGLA & FRECUENCIA \\
\hline $\begin{array}{l}\text { Adecuación de } \\
\text { evaluación }\end{array}$ & $\begin{array}{c}\text { Experiencia que señalan las profesoras como } \\
\text { acciones evaluativas para estudiantes NEE, que } \\
\text { involucren cambios en instrumento y tiempo de } \\
\text { evaluación. }\end{array}$ & AcAdEv & 7 \\
\hline $\begin{array}{l}\text { Evaluación } \\
\text { Personalizada }\end{array}$ & $\begin{array}{c}\text { Experiencia que señalan las profesoras como } \\
\text { acciones evaluativas para estudiantes NEE, } \\
\text { referida a la aplicación de un instrumento tipo } \\
\text { en forma individual, en un espacio exclusivo } \\
\text { para un alumno. }\end{array}$ & AcEvP & 2 \\
\hline $\begin{array}{l}\text { Adecuar según } \\
\text { las } \\
\text { características } \\
\text { del niño }\end{array}$ & $\begin{array}{l}\text { Experiencia en el proceso de evaluación de los } \\
\text { aprendizajes de un estudiante con NEE, } \\
\text { referido a realizar cambios en instrumentos o } \\
\text { tiempo en base a las características individuales. }\end{array}$ & $\mathrm{PrCN}$ & 4 \\
\hline
\end{tabular}

Fuente: Elaboración propia en base a 12 casos.

Con el objeto de aportar antecedentes en los párrafos siguientes se presentan las citas más significativas:

Realizar adecuación de la evaluación conforme a las características de cada niño o a los informes que presenta cada niño. (Entrevista 1)

Ella realiza algunas acciones como adecuaciones de acceso (...) realiza adecuaciones dependiendo de las necesidades que presenta el estudiante. (Entrevista 2)

Se pueden considerar ciertas... adecuaciones, eeh... a las evaluaciones. (Entrevista 6)

La adecuación y más que nada, lo que tiene que ver con los ítems, o sea hacerlo más entendible, más gráfico. (Entrevista 7)

Las evaluaciones, eeh, por lo general, están adecuadas en base a la necesidad del niño. (Entrevista 8)

También se le hacen adecuaciones, de acuerdo a las necesidades que tengan. (Entrevista 9)

Conforme a las características de cada niño o a los informes que presenta cada niño. (Entrevista 1)

La actividad, que el problema, el ejercicio sea más eeh... enfocado a lo que él sepa. (Entrevista 7)

Por lo general, están adecuadas en base a la necesidad del niño (...) del diagnóstico del chiquillo. (Entrevista 8)

Va a depender de las características del alumno, eeh... de acuerdo a su diagnóstico, obviamente, va ser diferente. (Entrevista 9)

Se observa que 5 de las 8 profesoras de educación básica, describen realizar un trabajo conjunto con profesora de educación diferencial y tomar decisiones conjuntas para la evaluación de los estudiantes PIE. Sobre los procedimientos de evaluación, 7 de las 8 profesoras de educación básica, hacen referencia a las pruebas escritas; pero también indican realizar procedimientos evaluativos que denominan "más concretos", entre los que 
mencionan rúbricas, pautas, trabajos grupales; desprendiéndose de esta información que si bien se prioriza el uso de la prueba escrita, las docentes entrevistadas tienen conocimiento de otros instrumentos y reconocen la necesidad de utilizarlos con sus estudiantes con NEE.

A continuación, se presentan citas que ilustran lo anteriormente planteado:

La coordinación colaborativa que tenemos con la profesora diferencial. Porque hay una retroalimentación con lo que sucede, con lo que ella trabaja cando sale... saca a los niños de aula, entonces ahi se genera el trabajo colaborativo entre... entre pares. (Entrevista 2)

Bueno, primero que nada, sería el trabajo que se realiza con la profesora especialista. (Entrevista 4)

En este caso, particularmente es la planificación en conjunto con la profesora diferencial... ese es mi mayor apoyo en este momento. (Entrevista 7)

Bueno, primero trabajo con especialista; que el trabajo con especialista no es algo aislado, por lo menos en mi centro de trabajo... eh, contamos con reuniones semanales para poder planificar en conjunto; los tiempos en que vamos a... a... permanecer juntas en el aula, son planificados y...el... conocimiento que la especialista también tiene de los diagnósticos de los niños, también es acabado. (Entrevista 8)

Sólo con la educadora diferencial, en este minuto. (Entrevista 11)

En relación con los instrumentos, la mayoría prioriza el uso de prueba escrita, pues los ajustes descritos guardan relación con ítems o textos incluidos en una prueba escrita; pero en el caso de las profesoras de escuela particular subvencionada (en adelante EPS), al parecer enfrentan mayor rigidez para decidir cómo evaluar a sus estudiantes, por lo que sus ajustes están relacionados con el acceso al instrumento de evaluación. Otro hallazgo importante es que las profesoras de escuela municipal (en adelante EM) parecieran tener mayor flexibilidad para decidir el tipo de ajustes a realizar; además, estas docentes pueden enfocarse en aspectos que no guardan directa relación con la calificación como evitar la frustración, las necesidades y progresos individuales, independiente del grupo curso al que pertenecen.

A continuación, se presentan citas que ilustran lo anteriormente planteado:

Eeh, una es el tiempo... para realizar... las adecuaciones al instrumento. (Entrevista 1)

Las barreras... lo que nos puede jugar en contra quizás, a nosotras, ipuede ser el tiempo! (Entrevista 3)

Eeh, el tiempo que uno trabaja con los niños; para mí es muy poco el tiempo. (Entrevista 12)

Frente a la justificación a las adecuaciones del instrumento de evaluación, esta se fundamenta ante la posibilidad de fracaso o se basan en fracasos antiguos; en ningún caso se argumenta en base a un motivo pedagógico o a las características neurológicas o sociales del niño, transparentando el motivo de la decisión.

Se explicó a las docentes que uno de los hallazgos de la fase cuantitativa, consistió en que los profesores priorizan el uso de los instrumentos prueba escrita para evaluar a sus estudiantes NEE. Frente a la consulta de por qué esos otros profesores priorizan la prueba escrita, se observa que cinco de las docentes considera que el tiempo es el principal factor externo que obstaculiza el desarrollo de otros instrumentos, cruzado con factores como la cantidad de niños en aula, las características físicas del aula o escuela. Las otras seis 
docentes consultadas lo atribuyen a factores internos del profesorado, como la comodidad ante un instrumento conocido, el poco conocimiento para elaborar otros y el mayor esfuerzo involucrado en el desarrollo de otras actividades evaluativas.

Presentamos citas que ilustran lo anteriormente planteado:

Que a veces por tiempo no nos alcanzamos a reunir, entonces, quizá sería... o sea, nos reunimos, pero nos reunimos después de la evaluación, entonces es complejo, porque no logramos coordinar anticipadamente con la educadora diferencial. (Entrevista 6)

Siete de las docentes consultadas atribuyen al trabajo de coordinación entre profesionales (profesor de aula, profesora diferencial, asistentes de aula) el principal facilitador para la adaptación de la evaluación. También se observa que algunas docentes de educación básica atribuyen a las profesoras diferenciales el conocimiento completo de los estudiantes NEE, manifestando que dependen de ellas.

Con el equipo PIE, se trabaja 3 veces a la semana... con educadora diferencial. Se trabaja sí con el curso completo, o sea no se trabaja solamente con los chicos que tienen necesidades educativas. Sí hay un trabajo... en sala, digamos, en la ubicación que nosotros hacemos de sala... se va trabajando sí, con los chicos que tienen necesidades educativas, como más personalizado; pero las actividades que se realizan en sí con la educadora diferencial es para el curso completo. ¡Las de evaluación! Al momento de evaluar... no hay evaluación diferenciada (...) Eeh... Es que, de las necesidades educativas que tenemos... específicas, en este caso de Gabriel, por ejemplo, que son de lenguaje. $\Upsilon$ con... Renato y Florencia, se trabaja más que nada personalizado. Las evaluaciones igual son más personalizadas con ellos dos. (Entrevista 10)

Con respecto a la principal barrera para la adaptación de la evaluación, un primer grupo de seis docentes hace alusión al tiempo, referido al envío de instrumentos evaluativos y sus ajustes, al tiempo de aplicación de una evaluación, al tiempo de desarrollo de una clase para alcanzar a realizar su respectivo cierre, al tiempo para realizar coordinación efectiva con profesora diferencial. Un segundo grupo de tres docentes expone respuestas que tienen relación con las exigencias y requerimientos solicitados por el establecimiento, los que dificultan la toma de decisiones sustanciales en torno a la evaluación; es decir que sólo se les permite realizar pequeñas modificaciones, porque deben cumplir con una estructura de prueba o un tipo de clase estándar.

Presentamos citas que ilustran lo anteriormente planteado:

Eeh... los tiempos en las que ellas envían esa evaluación... eeh, por ejemplo, si me la envían un día antes, obviamente, no es lo mismo que si lo envían dos o tres días antes. Cuando uno tiene más plazo, puede hacer consultas a la misma profesora respecto a algún ítem, o llegar a algún consenso si es que yo... eem... si tienen alguna sugerencia. (Entrevista 1)

Las barreras... lo que nos puede jugar en contra quizás, a nosotras, ;puede ser el tiempo! Eso sería una barrera, pero más... para nosotras... (¿̇Por qué sería?) Porque las actividades acá duran 40 minutos... en comparación al... programa de básica. Entonces son 40 minutos que se dividen en inicio, desarrollo y cierre. $\Upsilon$ un niño que tiene necesidades educativas, tener que presionarlo un poco para que avance y logre terminar dentro del tiempo... entonces por eso nos juega en contra. (Entrevista 3)

Que a veces por tiempo no nos alcanzamos a reunir, entonces, quizá sería... o sea, nos reunimos, pero nos reunimos después de la evaluación, entonces es complejo, porque no logramos coordinar anticipadamente con la educadora diferencial. (Entrevista 6)

En relación al significado que las docentes asignan a la participación de la familia en las decisiones de evaluación, las docentes valorizan el conocimiento que los apoderados tienen de sus hijos; otras entregan importancia a las estrategias que utilicen las familias, las que 
podrían validar dentro del trabajo de aula; un último grupo otorga importancia al acompañamiento del estudio que realizan las familias, dando a entender que algunas sí realizan un apoyo sistemático del proceso enseñanza-aprendizaje. No obstante, como obstaculizadores, está el poco conocimiento pedagógico que se atribuye a los padres y las conductas de negligencia o desinterés.

Presentamos citas que ilustran lo anteriormente planteado:

To creo que tendrían harto que aportar. Porque, en la mayoría de los casos, los chicos... O
en algunos casos, los chicos... funcionan de manera distinta en casa, que en el hogar; por lo
tanto, nos podrían aportar desde los intereses de los chicos... desde... em... Qué es lo que a
ellos les resulta más fácily más cómodo hacer o... cómo estudian, por ejemplo y... eso aplicarlo
aqui en el establecimiento. (Entrevista 1)

La familia también puede tener una visión... distinta a la que nosotros tenemos dentro del aula. El niño se comporta de manera distinta en su casa...que en el, que en el colegio (...) a las rutinas de aprendizaje o a las rutinas de trabajo que podría tener en la casa. (Entrevista 2)

Les podría beneficiar porque la familia... eh... tiene una mirada quizás un poco distinta sobre el estudiante, tiene una mirada desde el hogar, y a veces tenemos estudiantes que tienen comportamientos diferentes, tanto en el hogar como en el colegio; entonces, quizás pueda aportar con respecto a lo que ellos conozcan sobre sus gustos, sobre las dificultades que van observando al momento de estudiar. (Entrevista 6)

Emm... ayudaría a ... a conocer más las características del alumno, en donde ve la familia que se puede desempeñar mejor... eh... porque lo conocen más y saben que... qué cosas él... a qué puede responder. (Entrevista 9)

\section{Discusión y conclusiones}

\subsection{Acciones para la adaptación evaluativa}

En relación a las acciones de adaptación evaluativa (cómo lo realizan) y las adaptaciones curriculares (qué realizan) que se esperaba encontrar, los hallazgos de la fase cuantitativa, evidencian incoherencias en función a los conceptos que declaran los profesores y las acciones que describen realizar (Marfán et al., 2013).

Los profesores indican que se centran en construir aprendizajes nuevos en torno a los aprendizajes significativos de los estudiantes, pero no priorizan ningún momento o una intencionalidad de la evaluación y esto no es coherente con la postura de aprendizaje constructivista declarada, que prioriza la evaluación procesual-formativa; utilizan instrumentos tradicionales de evaluación (San Martín, 2012); no mencionan priorizar o ajustar objetivos en relación a características individuales (Gimeno y Pérez, 1992), por el contrario, las profesoras de colegio particular subvencionado expresan exigencias y requerimientos del establecimiento que dificultan la toma de decisiones.

\subsection{Vinculación entre las adaptaciones evaluativas y la normativa vigente}

Dado que la normativa vigente para PIE entró en vigencia el año 2009, se esperaba que los profesores manejaran conceptos y realizaran acciones coherentes a la normativa vigente. Los docentes señalan evaluar los aprendizajes de los estudiantes considerando equitativamente los cuatro elementos sugeridos por la normativa: (a) aprendizajes logrados, (b) estilo de aprendizaje y motivación, (c) aspectos del desarrollo personal y 
social, (d) capacidades fortalezas y dificultades (MINEDUC, 2013). Sin embargo, declaran que no se cumple la estrategia DUA de proporcionar múltiples medios de compromiso ofreciendo opciones que reflejen los intereses de los alumnos, la que se relaciona con el desarrollo de la autonomía, la motivación y el clima de aceptación en el aula. Además, se encuentran en una fase inicial de implementación de la inclusión, correspondiente a la organización de un grupo de coordinación (Marfán et al., 2013).

\subsection{Intervención del equipo multiprofesional}

No se detecta coherencia entre las sugerencias de la normativa y los agentes involucrados en la planificación, puesto que se debe incorporar a la família (MINEDUC, 2013). Los docentes de aula y profesores diferenciales trabajan en conjunto, eventualmente con fonoaudiólogo o psicólogo, pero no consideran otros integrantes en su equipo multiprofesional, que pueda trabajar colaborativamente para mejorar la calidad de los aprendizajes (Duk y Loren, 2010) y respetar las diferencias individuales de los estudiantes PIE. Se confirma que las decisiones sobre evaluación, son determinadas solamente entre profesoras de aula común y profesoras diferenciales (López et al., 2014) en conjunto, desestimando la participación de otros.

\subsection{Experiencias de ajuste evaluativo}

La evaluación diferenciada es la principal acción realizada por los docentes. Se señala el desarrollo de un trabajo con profesora diferencial para tomar las decisiones y se describen ajustes a la evaluación en su formato, simplificación de ítems y ajustes de tiempo para la aplicación de un instrumento escrito (San Martín, 2012).

\subsection{Facilitadores y obstáculos para la adaptación de la evaluación}

Para conocer la causa de las incoherencias encontradas, se consultó por los facilitadores y obstaculizadores para realizar el proceso de la adaptación de la evaluación para sus estudiantes NEE. Entre los principales facilitadores declarados, se encuentra (a) trabajo de coordinación entre profesionales (López et al., 2014; MINEDUC, 2013), (b) diálogo para tomar decisiones en torno a las necesidades de sus estudiantes (Ahumada, 2005; MINEDUC, 2013) y (c) existencia de material específico y concreto, que es utilizado para la evaluación (Decreto exento $\left.n^{\circ} 83,2015\right)$.

Entre los obstaculizadores se encuentra (a) el tiempo, referido al envío de instrumentos evaluativos y sus ajustes, la aplicación de una evaluación, el desarrollo de una clase para alcanzar a realizar su respectivo cierre, al tiempo para realizar coordinación efectiva con profesora diferencial (López et al., 2014); y (b) las exigencias y requerimientos solicitados por el establecimiento, que dificultan la toma de decisiones (López et al., 2014).

\subsection{Facilitadores y obstáculos para la participación de la família}

Los facilitadores para participación de la familia son el (a) conocimiento que los apoderados tienen de sus hijos; las (b) estrategias que utilizan las familias dentro del hogar, al desarrollar actividades de enseñanza-aprendizaje, las que podrían validarse dentro del trabajo de aula; y el (c) acompañamiento del estudio que realizan las familias. Los obstaculizadores descritos, son la (a) baja preparación académica que se atribuye a los padres y su correspondiente baja capacidad de apoyar u opinar sobre lo pedagógico (López et al., 2014) y las (b) conductas de negligencia o desinterés en participar del proceso de enseñanza-aprendizaje de sus hijos. 


\section{Referencias}

Ahumada, P. (2001). La evaluación en una concepción de aprendizaje significativo. Valparaiso: Ediciones Universitarias de Valparaíso.

Ahumada, P. (2005). Hacia una evaluación auténtica del aprendizaje. Ciudad de México: Paidós.

Bericat, E. (1998). La integración de los métodos cuantitativo y cualitativo en la investigación social: significado y medida. Barcelona: Ariel.

Biblioteca del Congreso Nacional de Chile. (2010). Historia de la Ley 20.422. Santiago de Chile: Diario Oficial.

Buendía, L., Colas, P. y Hernández, F. (1998). Métodos de Investigación en Psicopedagogía. Madrid: McGraw-Hill.

Castejón, J. L. y Navas, L. (2009). Unas bases psicológicas de la educación especial.. Madrid: Editorial Club Universitario.

Decreto ${ }^{\circ}$ 1. (1998). Reglamenta Capítulo II Título IV de la ley 19.284 que establece normas para la integración social de personas con discapacidad Chile. Retrieved from http://www.leychile.cl/N?i=155849yf=2000-02-1 1yp

Decreto supremo $\mathrm{n}^{\circ} 170$ (2009). Decreto con toma de razón $N^{\circ}$ O170. Fija normas para determinar los alumnos con NEE que serán beneficiarios de las subvenciones para educación especial Chile. Recuperado de https://especial.mineduc.cl/wp-content/uploads/sites/31/2018/06/ DTO-170_21-ABR-2010.pdf

Decreto exento $\mathrm{n}^{\circ}$ 83. (2015). Aprueba criterios y orientaciones de adecuación curricular para estudiantes con NEE de educación parvularia y educación básica. Recuperado de http://especial.mineduc.cl/wp-content/uploads/sites/31/2016/o8/Decreto-83-2015.pdf

Duk, C. y Loren, C. (2010). Flexibilización del Currículum para Atender la Diversidad. Revista Latinoamericana de Inclusión Educativa, 4(1), 187-210.

Echeita, G. (2006). Educación para la inclusión o educación sin exclusiones. Madrid: Narcea.

Gimeno, J. y Pérez, A. I. (1992). Comprender y Transformar la Enseñanza. En A. I. Pérez Gómez y J. Gimeno Sacristán (Eds.), Comprender y Transformar la Enseñanza (pp. 34-62). Madrid: Morata. https://doi.org/10.1017/CBO9781107415324.004

Hernández, R., Fernández, C. y Baptista, M. (2014). Metodología de la Investigación. Madrid: McGraw-Hill.

Ley 20.201. (2007). Modifica el $\mathrm{DFL} n^{\circ} 2$, de 1998, de educación, sobre subvenciones a establecimientos educacionales y otros cuerpos legales. Santiago de Chile: Biblioteca del Congreso Nacional.

Ley 20.370. (2009). Establece la ley general de educación. Chile. Recuperado de https://www.leychile.cl/N?i=1006043yf=2009-09-12yp=

Ley 20.422. (2010). Establece normas sobre igualdad de oportunidades e inclusión social de personas con discapacidad. Recuperado de https://www.leychile.cl/N?i=1010903yf=2016-06-28yp=

Llanos, A. y Escamilla, E. (1995). La evaluación del aprendizaje y de la enseñanza en el aula. Madrid: Edelvives. 
López, V., Julio, C., Pérez, M. V., Morales, M. y Rojas, C. (2014). Barreras culturales para la inclusión: políticas y prácticas de integración en Chile. Revista de Educación, 363(1), 256-281. https://doi.org/10.4438/1988-592X-RE-2012-363-180

Marfán, J., Castillo, P., González, R. y Ferreira, I. (2013). Informe Final de Estudio : estudiantes con necesidades educativas. Recuperado de http://www.mineduc.cl/usuarios/edu.especial/doc/201402101719500.InformeEstudioIm plementacionPIE2013.pdf

Ministerio de Educación de Chile. (2013). Orientaciones técnicas para programas de integración escolar (PIE). Santiago de Chile: Ministerio de Educación de Chile.

Muñoz, E. (2006). Talleres para la construcción de instrumentos evaluativos en educación. Santiago de Chile: Bibliográfica Internacional.

Organización Mundial de la Salud. (2001). Clasificación internacional del funcionamiento, de la discapacidad y de la salud. Recuperado de

http://www.imserso.es/InterPresent2/groups/imserso/documents/binario/435cif.pdf

Peña, M. (2013). Análisis crítico de discurso del decreto 170 de subvención diferenciada para necesidades educativas especiales: El diagnóstico como herramienta de gestión. Psicoperspectivas, 12(2), 93-103.

Pérez, G. (2011). Investigación cualitativa. Retos e interrogantes. II Técnicas y análisis de datos. Madrid: La Muralla.

San Martín, C. (2012). Atención a la diversidad en el contexto educativo chileno: concepciones del profesorado sobre evaluación y diseño de la propuesta curricular. REICE. Revista Iberoamericana sobre Calidad, Eficacia y Cambio en Educación, 10(4), 165-183.

\section{Breve CV de las autoras}

\section{Karla Muñoz Durán}

Profesora de Educación Diferencial, coordinadora del Proyecto de integración escolar de la Comuna de Hualpén, región del Bio Bio, Chile Magíster en Ciencias de la Educación, mención evaluación de la Universidad Católica de la Santísima Concepción, Chile. ORCID ID: 0000-0001-5608-1135. Email: kmunoz@magisteredu.ucsc.cl

\section{Maite Otondo Briceño}

Académica asistente del Departamento de Fundamentos de la Pedagogía de la Universidad Católica de la Santísima Concepción, Chile. Magíster en Ciencias de la Educación mención Currículum Universidad Metropolitana de Ciencias de la Educación, Magíster en Gestión Universidad del Desarrollo, Doctoranda en Ciencias de la Educación Universidad de Sevilla España. Capítulo libro: Formación de profesores diferenciales: Desde la mirada de las Universidades pertenecientes al CRUCH", libro "Educación Inclusiva en Latinoamerica: Políticas, Investigaciones y Experiencias", Editoriales CAPUB (México) e INTERTEXTO (BRASIL), 2016. Capítulo libro "La retroalimentación como recurso de autorregulación de los aprendizajes y regulación de la docencia”. Propiedad intelectual $\mathrm{N}^{\circ}$ : 268.881 Obra Literaria (escrito) titulada: Cuestionario: Escala de impacto de la retroalimentación, 2016. Artículo: Políticas Públicas, Procesos Formativos: Publicado: "Investigaciones, experiencias didácticas e innovaciones pedagógicas en la formación inicial docente de la Universidad de 
Concepción", 2016. Artículo: Co docencia en alumnos de Pedagogía en Educación Diferencial de la Ucsc Revista Rexe. Proyectos: DIN 2015 La inclusión en Educación Superior de estudiantes con discapacidad en la UCSC. Proyecto FAD 2017 Diseño Universal de Aprendizaje para dar respuestas pertinentes a la diversidad individual de aprendizaje en carreras de pedagogía de la Facultad de Educación de la UCSC. ORCID ID: 0000-0001-9513-3794. Email: maite@ucsc.cl 